\title{
THE RESEARCH DOMAIN OF PRODUCT-SERVICE SYSTEMS AND VOICE OF THE CUSTOMER: A SYSTEMATIC MAPPING
}

\author{
Toller, Carl Nils Konrad; \\ Bertoni, Marco \\ Blekinge Institute of Technology
}

\begin{abstract}
Product-Service Systems (PSS) have emerged as a key concept to meet the societal and market trends of increasing customer needs through the entire life-cycle. Unfortunately, several companies are struggling with getting revenues from service investments and translating 'real needs' to design improvements. The demand of the designer to go beyond the Voice of the Customer (VoC) is evident. This paper aims to map the interventions proposed by research in the area of PSS and VoC. Using a systematic mapping approach, the research domain was analyzed with regards to context and interventions. The results show a progressive development in the research area with a focus on the specification and realization of needs. A gap exists in connecting the engineers with 'real needs' and integrating the customer as a natural part of the entire development cycle of a PSS. By performing a systematic mapping, future research can be more focused and hopefully increasing its impact.
\end{abstract}

Keywords: Product-Service Systems (PSS), Research methodologies and methods, Design management, Voice of the Customer, Systematic Mapping

\section{Contact:}

Toller, Carl Nils Konrad

Blekinge Institute of Technology

Mechanical Engineering

Sweden

carl.toller@bth.se 


\section{INTRODUCTION}

Recent societal and market trends have brought to the emergence of business propositions that mix and match hardware, software, and service aspects to cope with increasingly sophisticated customer expectations and needs. Many companies in recent years have spotlighted the opportunity of exploiting such a 'servitization' strategy in manufacturing and of using value as a driver for making early design decisions (Baines et al., 2009). As discussed by Kuijken et al. (2017), while product manufacturers are increasingly attracted by the opportunity of engaging in servitization initiatives, a great deal of investments is currently dedicated to developing integrated offerings in form of Product-Service Systems (PSS) (Tukker and Tischner, 2017). Yet, despite several successful examples, the servitization transition comes with considerable challenges. In 2011, Neely et al. (2011) conducted an extensive analysis of 12,521 companies and found out that servitized firms tend to generate lower net profit compared to pure manufacturing ones. These companies are often not able to cover servitization investments, a phenomenon that was already spotlighted in the literature and named the "service paradox" (Gebauer et al., 2005). The paradox describes a situation in which a company that invests heavily in extending its service business is not able to achieve correspondingly higher returns. As explained by Cavalieri and Pezzotta (2012), PSS development raises new issues and invites the designers to go beyond the Voice of the Customer (VoC) to know more about the 'real needs' for the 'solution'. These include, for instance, the individual's emotional state of mind, as well as his/her perceptions/preconceptions in a situated and changeable context. Traditionally, engineers have focused on meeting functionality and cost objectives linked to the realization of a physical artifact (Ulrich and Eppinger, 2011). The transition towards servitized business models stresses the need to embrace a more holistic approach and to deal with the issue of designing 'goodness' (Cheung et al., 2012), and of initiating the development work from the standpoint of how to add value to customers and the stakeholders (Lemon and Coley, 2008) along the life cycle.

\section{MOTIVATION AND OBJECTIVES}

The bundling of services with product offerings does not always render an improvement in business performances. The root-cause of this phenomenon is sometimes found in the inability to model customer expectations and needs in the early stages of PSS development. This is explained, see Sotelo et al. (2018), as the misalignment between the actual stakeholder needs (and the quality characteristics that the stakeholders want) versus the list of needs and expectations retained by designers along the development process. Faisandier (2012) pinpoints that the continuity between the needs, their translation into technical requirements, and the design of potential solutions is not always ensured along the systems engineering cycle. As a result, systems (such as the PSS) are not able to meet the purpose for which they are conceived and designed. Faisandier (2012) further proposes a model named the Cycle of Needs, which describes how the 'real' needs of the stakeholders are systematically translated into the requirements of the solution. Improving the efficiency and the effectiveness of this 'translation' is a difficult but strategic issue for the development of PSS solutions.

The main aim of this paper is to spotlight the 'interventions', as defined by Petersen et al. (2008), currently proposed in the PSS literature when facing the issue of 'tuning in' to the VoC for PSS. The paper gathers and categorizes available evidence relating to how 'real needs', in the way expressed by Faisandier (2012), are captured, filtered, prioritized, and analyzed to support the development of PSS able to 'add value' in a given market and business situations. Noticeably, the paper does not target a specific research question as done in systematic reviews. Rather, it borrows the concept of systematic mapping from social sciences research to build a classification scheme and to structure the field of interest. In turn, the objective of the study is to provide an overview of the research area at the intersection of VoC and PSS. Firstly, the paper maps the frequency of publication over time in the domain to spotlight any significant trend. Secondly, it identifies the quantity and type of research and results available within it, to reveal the knowledge domains that have been emphasized in past research, and to identify gaps and opportunities for future research, paving the ground for the definition of more specific research questions. The results are presented both in the form of summary statistics - e.g. showing the number and type of publications per year - and in form of a bubble plot that counts the number of publications in the VoC/PSS domain versus the type of intervention proposed. 
While section 3 illustrates the Cycle of Needs model, section 4 describes in detail the systematic mapping process and section 5 presents the results of the analysis. The authors further elaborate on the findings in section 6 and conclusions are drawn in section 7.

\section{THEORETICAL FRAMEWORK - THE CYCLE OF NEEDS MODEL}

According to Faisandier (2012), a need can have different levels of maturity, progressing from real needs to realized ones. This maturity process is illustrated in Figure 1. In the proposed model, the upper needs are subject to market analysis, while the lower ones are screwed toward the implementation of a 'system'.

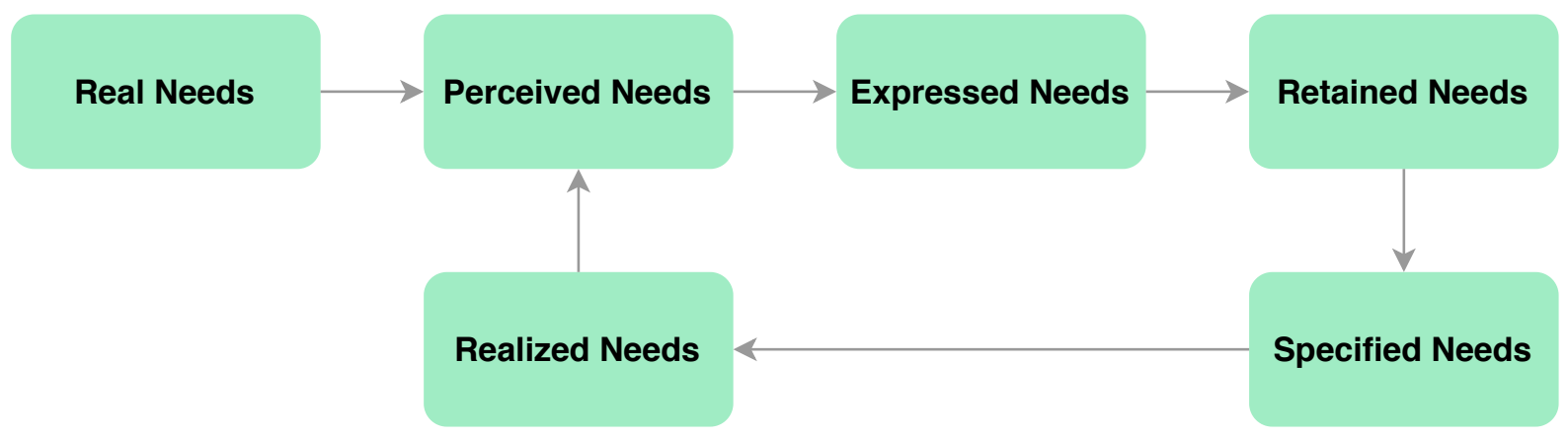

Figure 1. Cycle of Needs, based on Faisandier (2012, p. 82)

At the start, Real Needs represent the entirety of tacit and explicit needs a customer and/or stakeholder may be aware of. Noticeably, real needs are 'raw data' and represent the entire need space for a specific context. Once an individual or an organization becomes aware that a set of needs exist, those are transformed into a Perceived Needs. These are based on a person's or group's perception that something is wrong, that there is a lack of something, or that there is something that could improve a given situation. Perceived needs are often presented as a list of organized expectations resulting from an analysis of the usage conditions for the considered action. When individuals or organizations can express, organize and weigh the perceived needs internally, these are transformed into Expressed Needs. The latter is articulated in form of generic actions or constraints and is typically prioritized from the customer's viewpoint. For instance, if safety is a primary concern for an electric vehicle, the expressed need to "protect the machine operator against accidental shorting" may be prioritized over other expressed needs such as "ensure the ergonomic position of the operator".

Retained Needs are a subset of the expressed needs that represent the ones selected by the provider to guide later development activities. Not all expressed needs can be retained, mainly because these are often overlapping, contradictory, and differ in terms of granularity. Only those needs that are 'retained' by the design team will be later used as a base for developing a solution and defining its requirements. Retained needs are then further refined and translated to represent the problem from the viewpoint of the solution provider, generating the so-called Specified Needs. Eventually, these needs are actualized through the design and development of a solution, becoming the carrier of Realized Needs. These are the needs provided to the customer and ultimately compared to the perceived needs. Any potential misalignment is then fed back into the loop for further iterations and development. The misalignment can also work as a tool for increasing the understanding of the real needs.

\section{SYSTEMATIC MAPPING PROCESS}

The systematic mapping process is adapted from the methodology presented by Petersen et al. (2008). The key goal is to map the current and previous research in the desired topic (or research domain) and to categorize these along two dimensions; context and intervention. The process is illustrated in Figure 2, which shows the main steps of the approach at the top and the generated output at the bottom. There are some differences between this process and the one proposed by Petersen et al. (2008). Firstly, the step dedicated to the "Definition of Research Question" is named "Definition of Research Domain" to better reflect the actual activity taking place in the mapping. Furthermore, the context facet is synthesized by the definition of the research domain, and it is not a result of key-wording. 


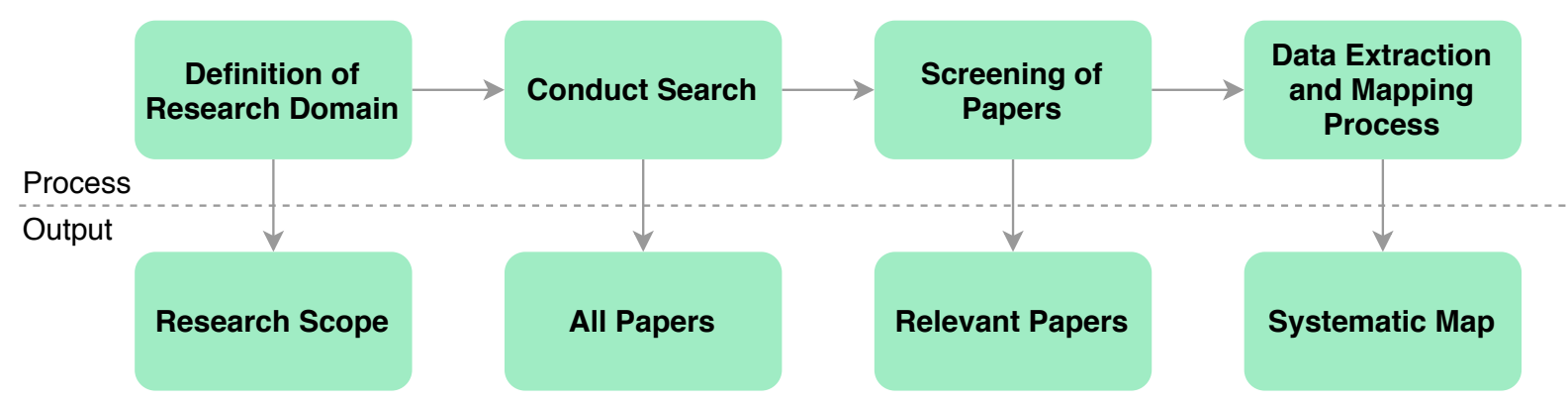

Figure 2. Systematic mapping process, inspired by Petersen et al. (2008)

Figure 2 features 4 main steps, which are Definition of Research Domain, Conduct Search, Screening of Papers, and Data Extraction and Mapping Process. Noticeably, when kicking-off a systematic mapping study one shall consider that the research domain needs to be narrow enough for the mapping process to be manageable. In this case, the authors only considered those contributions at the intersection between the PSS domain and the realm of VoC research.

The second step is dedicated to the definition of a relevant search string at such an intersection. The string was generated by the authors through different iterations, having the care to include all relevant synonyms for PSS and VoC identified in the literature. In its final version, the string featured 12 synonyms for PSS and 18 synonyms for VoC, like the following:

TITLE-ABS-KEY ( "product-service system" OR "product-service bundle" oR "integrated

product service" OR "industrial product service" OR "product-service offer*" OR

"integrated solution" OR "total care product" OR "hybrid offer*" OR serviti?ation

OR "functional product" OR "service paradox" OR "outcome-driven innovation")

AND ("voice of the customer" OR "customer need" OR "consumer need" OR "customer

expectation" OR "consumer expectation" OR "customer preference" OR "consumer

preference" OR "customer requirement" OR "consumer requirement" OR needfinding OR

"customer mapping" OR "consumer mapping" OR "customer involvement" oR "consumer

involvement" OR "customer integration" OR "consumer integration" OR "customer

insight" OR "consumer insight")) AND (LIMIT-TO(DOCTYPE, "CP") OR LIMIT-TO(DOCTYPE, "ar"))

The SCOPUS database was later accessed to retrieve all those contributions answering to the string in the title, abstract, and keywords. As a publication-based filter, only journal articles and conference proceedings were considered for further analysis. Eventually, the search - which was performed on December 2nd, 2020 - rendered a list of exactly 400 hits. All the duplicates were initially removed, together with all contributions not written in English. Later, the authors defined content-based inclusion criteria in several iterations, to mitigate the risk for personal biases to affect the screening activity. The following criteria were then applied to evaluate the suitability of each retrieved item for the scope of the study:

- the notion of PSS lies at the core of the paper, and is not merely discussed marginally;

- the notions of $\mathrm{VoC}$ and customer satisfaction lie at the core of the paper, and are not merely discussed marginally;

- the paper proposes an 'intervention', typically in form of either a process, a method, a model, a tool, or a case.

- $\quad$ one or more steps of the Cycle of Needs model are directly or indirectly discussed in the paper; or

- the paper discussed the process of 'needfinding' and 'requirements definition' from a contextual perspective, without targeting any specific step of the Cycle of Needs model;

The number of papers retained for further analysis was reduced to 143 , of which 66 journal articles and 77 conference proceedings. These were further analyzed and categorized based on the specific intervention being proposed. For the intervention facet, the same categorization proposed by Petersen et al. (2008) was used, except for the 'metric' facet being changed to 'case'. The argument for using this structure is that it promotes a clearer understanding of where the research domain has focused and what values can be drawn from it. Noticeably, a tool is defined in the paper as a device (e.g., software) that can carry out a particular function and that transforms an input into an output. A model is defined as an abstract representation of reality. While it does not necessarily perform the input-output transformation, a model is useful due to its explanatory and predictive power. Both descriptive and prescriptive models are considered in this categorization. Furthermore, a paper is considered to describe a 'case' only if it 
features the description of a real deployment and/or implementation, meaning that potential applications or hypothetical cases are not counted.

\section{RESULTS}

To visualize the findings from the systematic mapping, a bubble chart was constructed, as shown in Figure 3. The diagram is structured so that the horizontal axis represents the intervention proposed while the vertical axis represents the context, i.e. the steps of the Cycle of Needs model Faisandier (2012). The area of the bubble correlates to the number of papers found at each intersection.

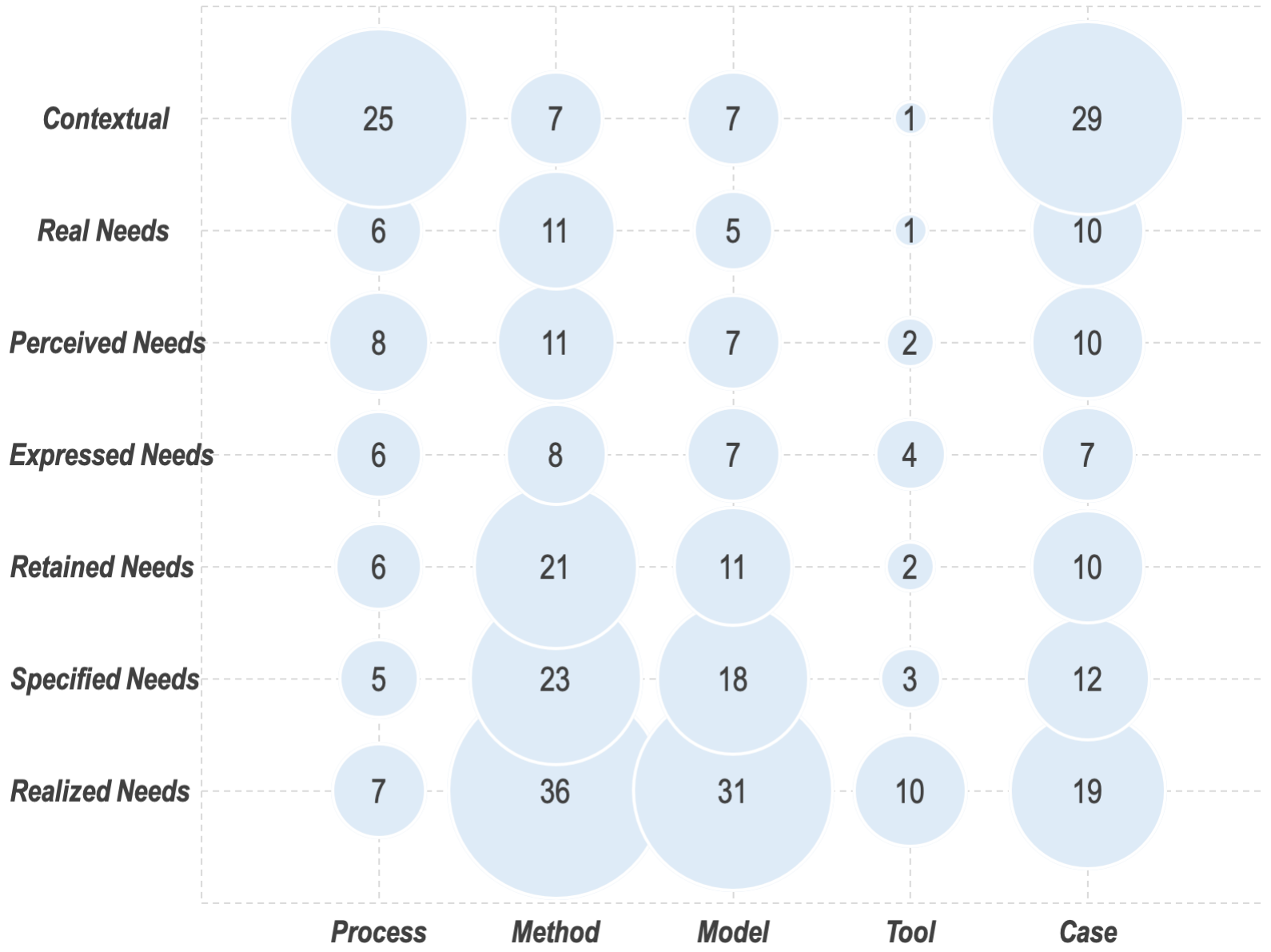

Figure 3. Bubble chart displaying the results of the systematic mapping study

A first remark is that the majority of papers are revolving around methods and models moving from retained to realized needs. These contributions analyze the 'need' issue with a focus on supporting companies in realizing artifacts and services that can raise the 'satisfaction of the acquirer' in the words of Faisandier (2012). Noticeably, the concept of 'realized needs' is proper of the solution domain. The focus lies on the results of the development work and the exploration of the solution space, rather than on the exploration of the broader problem space. What the bubble diagram reveals is also a seemingly weaker focus on the 'PSS development processes' issue. There is comparably little research that aims at defining and implementing the several steps needed to 'mature' the real needs and to understand how to support designers in increasing maturity. Furthermore, several contributions address the 'process' perspective without including explicitly aspects of 'needfinding', which are only described at a contextual level. The analysis further reveals that only a few papers propose 'interventions' in the form of tools to support the definition of the needs in the different stages of the Cycle.

It is also evident that the literature is comparably less rich when it comes to proposing tools to support the translation of real needs to realized needs. This lack of tools is particularly evident when looking at the initial stages of the progression. There is a perceived knowledge gap with regards to means that can raise people's and consumers' awareness that something is wrong or lacking. At the same time, little is said about design support tools that can raise awareness among consumers of their hidden 
and tacit needs, mainly with regards to improvements that could be made and opportunities that could be capitalized upon. This does, in turn, affect the ability to capture those tacit gains and pain points influencing the overall value of a solution. Furthermore, the literature in the PSS domain seems to lack specific tools to support the transition from retained to specified needs, coping with the problem of translating stakeholder statements (the ones that are retained by the solution provider, i.e., the retained needs) - that lack definition, analysis, and possibly consistency and feasibility - into something more structured and systematic, ready to be used in the engineering design process.

A significant part of the analyzed contributions focuses on contextual factors related to the development of processes to support the needfinding progression represented in the bubble chart. Furthermore, most of the literature does not propose a specific 'intervention' from the perspective of how to support the need progression and translation but is rather limited to the description and discussion of specific cases.

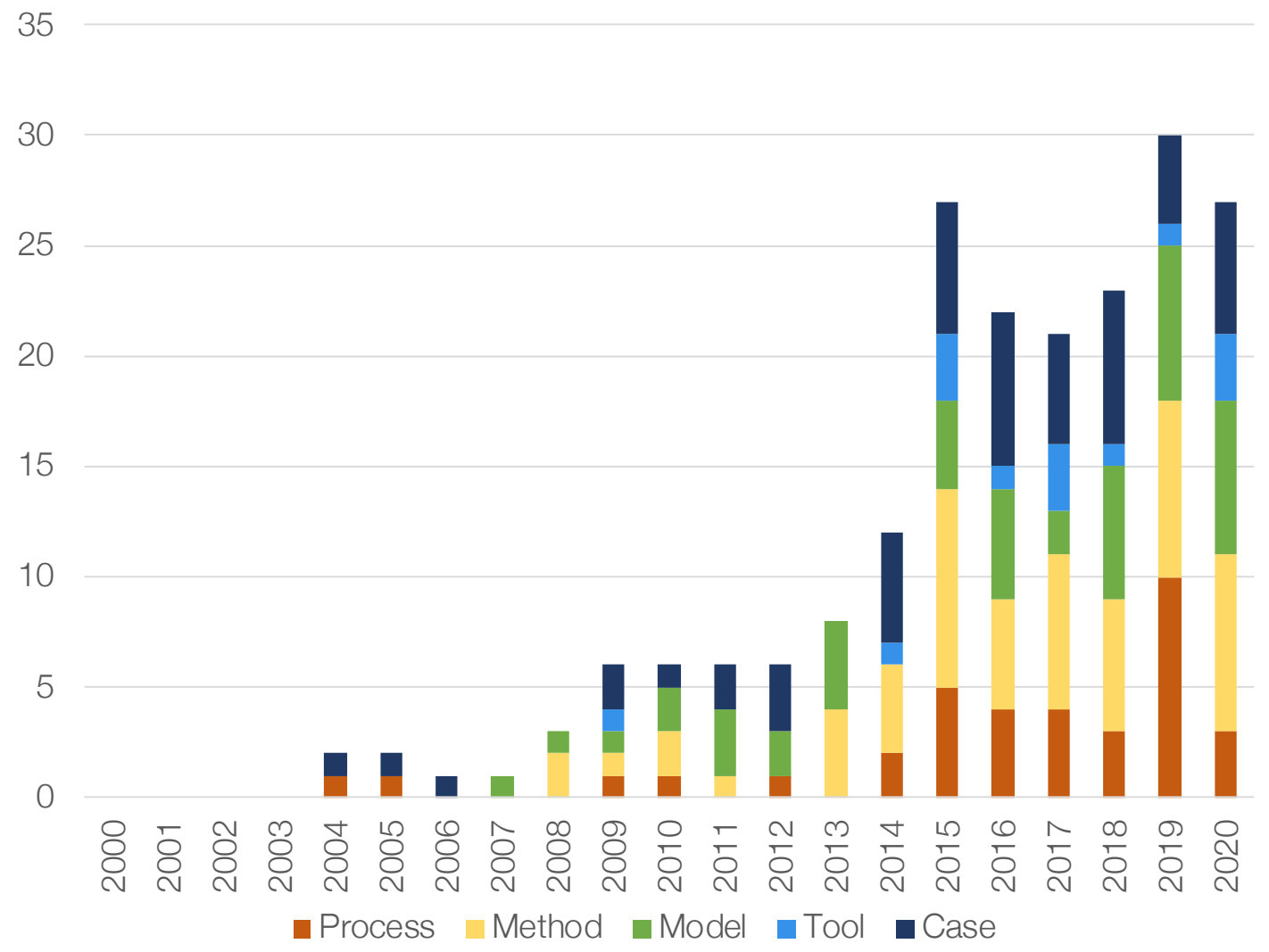

Figure 4. Intervention facet over time

By sorting the retained papers over time, it is possible to observe how the field has developed in the last 20 years, from 2000 to 2020. It is noticeable that the research at the PSS vs. VoC intersection has gradually developed, with a visible acceleration between 2013 and 2015. In the same way, it is possible to look at how the intervention and context facets have developed as a function of time. Figure 4 illustrates which type of interventions have been proposed over the years. The distribution is quite stable with a predominance in methods and models. It is also noticeable how in the past few years, the number of contributions focused on case applications has grown, often with the intent of validating different forms of intervention. Otherwise, no significant patterns are observed in Figure 4, and no specific intervention type seems to attract more interest - or to grow at a much higher pace - than others in recent years. The context facet and its development throughout the years is represented in Figure 5. Also here, the distribution has remained rather stable with a clear focus on realized needs which still today is the largest contribution. Noticeably, the research on real, perceived, and expressed needs has not been much in the spotlight in the last 20 years, while those contributions dealing with the more 'contextual' aspects of the PSS vs. VoC intersection have been expanding since 2014. 


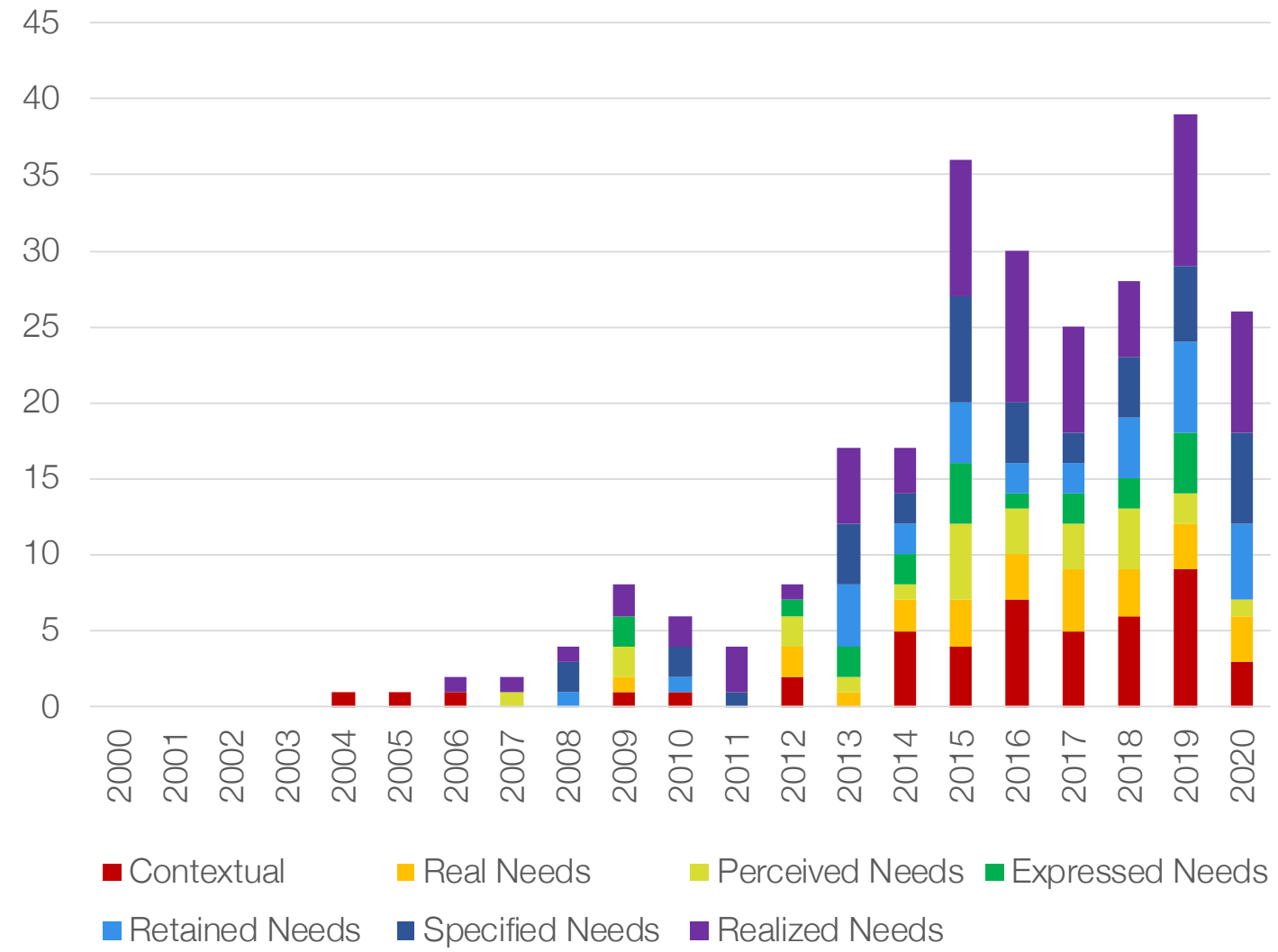

Figure 5. Context facet over time

\section{DIscussion}

The research at the intersection between PSS and $\mathrm{VoC}$ has attracted significant interest over the last years and appears to be evolving - with an increasing number of contributions being published in recent years across the spectrum of interventions and contexts highlighted in the study. Considering the current market trends, this research 'stream' is expected to continue growing along with the growth of servitized business models. The main benefit of performing a systematic mapping of this 'stream' - before attacking specific research questions through a systematic literature review - is found in the opportunity to obtain a broader picture of the current state of the academic research, to identify potential gaps across thematic areas. By remaining at a holistic level, it is possible to cover more effectively a larger number of papers in a shorter time frame compared to a systematic review, which is a more time-consuming task. Additionally, the mapping allows for easier communication of the research results and their impact, while it can be used by researchers to argue for future research strategies.

The mapping activity focused mainly on the notion of ' $\mathrm{VoC}$ ' in the context of PSS. For this reason, other fields such as system engineering, marketing, and 'traditional' product development were excluded. Most likely, many of the challenges and issues in VoC are shared across the domains as well as plausible interventions. Future research shall aim at learning more from these fields, cross-fertilizing the findings from the PSS realm with those that have not been considered in the current investigation. It is important to notice, however, that the implementation of such new findings is likely not to be straightforward without necessary adjustments.

The results of the systematic mapping activity show that a large portion of the papers is focusing on retained, specified, and realized needs. One plausible cause for this is that engineers usually do not work with raw data but with refined and processed data. The traditional way of working in silos can be a contributor to this, i.e. it is often common for manufacturing companies to leave the marketing department to collect and process the $\mathrm{VoC}$ before the list of needs for the solution is communicated to the designers. Research related to the 'engineering design' silo might thereby focus more on interventions applicable for that specific silo and to a lesser degree regard the entire cycle of needs. Another cause can be that those needs are already well-established in other research domains and can be directly translated to the domain of PSS/VoC and hence not been interesting for researchers. 
Faisandier (2012) makes clear in his analysis that real needs are hard to gather and even for the customer themselves to perceive. This can be an explanation for interventions directed to this type of need is not well established in the research field. The authors believe that it is worth considering studying this need deeper, and deploying interventions that can better translate and realize real needs. One suggested approach for this is data-driven design as it utilizes operational, context-aware data to describe the behavior and 'performance' of the deployed system. The high number of case studies correlated to this research domain indicates that the proposed interventions have been validated, increasing the quality of research.

Concluding the discussion above, some points and remarks for future research can be made. Firstly, it is of interest to perform a more in-depth systematic literature review on the $\mathrm{VoC}$ and needfinding process. The review should expand beyond the PSS context and look at other research fields to find similar challenges and proposed interventions. Hopefully, this can also increase the understanding of how VoC can be used by designers earlier and support the decision-making. Secondly, it is interesting to see how the topic of data-driven design can support VoC. Gathering and analyzing operational, contextaware data can increase the understanding of the PSS in use and provide additional insights on how the system provides value to the stakeholders. Finally, it could be valuable to see if any of the suggested interventions are predominated by the industry and how well the developed methods, models, and tools 'stick' in the longer perspective. This could contribute to a more efficient process for researchers and firms to determine correct interventions based on descriptive studies.

Looking at the mapping process itself, some challenges and aspects need to be highlighted. To reduce personal biases in the classification, inclusion, and exclusion criteria were made explicit from the start and continuously discussed in the team of authors. Furthermore, the use of well-established bibliographic databases mitigated the risk of including in the mapping research items of dubious quality. Noticeably, a common, unambiguous, and clear terminology across the analyzed contributions - as well as the lack of a homogeneous structure among the set of papers been reviewed - made it difficult to categorize them on several occasions. Furthermore, several papers span multiple categories, for instance proposing different intervention types (e.g., process and models), making the creation of the bubble chart to be much less straightforward. The same classification problem was observed when working with the Cycle of Needs model. For instance, it was not always easy to distinguish between expressed and retained needs in some of the shortlisted papers. This problem was mitigated mainly through discussion within and outside the team of authors to reflect on how and why a given contribution had to be categorized.

\section{CONCLUSIONS}

Product-Service Systems are becoming increasingly more important as a means to satisfy increasingly sophisticated customer needs and to create added value for users and other stakeholders. However, their development comes with a set of new challenges for the manufacturing firm. The creation of 'solutions' and not merely 'products' stresses the need to better understand the Voice-of-the-Customer, to ensure customer satisfaction and, ultimately, the market viability of what proposed. This paper has shown how the quantity and nature of the research effort spent at the intersection between Voc and PSS research tend to focus on a few themes and facets, leaving significant gaps with regards to the needed design support to guide the definition of the requirements of PSS embryos. The literature was found to mainly target the engineers' perspective for specifying and realizing the customer needs, where methods and models have been the dominant interventions. Moreover, there is a gap in the research domain with regards to the earliest stages of the Cycle of Needs model, with emphasis on models and tools able to raise awareness on the real needs for a solution before the definition of requirements takes place. Therefore, the PSS domain can benefit from studying VoC in adjacent research fields to find commonalities and deepen the domain of knowledge further. The final goal is to help manufacturing firms in working with VoC for PSS over departmental borders and at a more strategic level. It is also interesting to see if the emergence of data-driven design can support the understanding of the PSS operational behavior and how it compares to the VoC. Connecting how the user operates the PSS with how the customer perceives the PSS can hopefully simplify the needs process and validation of the VoC. Finally, a comparison study of methods, models, and tools proposed in the mapping compared to what is used by the industry based 
on descriptive and prescriptive studies can aid researchers and firms to more efficiently determine good interventions to experienced issues.

\section{ACKNOWLEDGEMENTS}

The research leading to these results has received financial support from the Swedish Knowledge and Competence Development Foundation (Stiftelsen för kunskaps- och kompetensutveckling) through the Model Driven Development and Decision Support research profile at Blekinge Institute of Technology.

\section{REFERENCES}

Baines, T., Lightfoot, H., Benedettini, O. and Kay, J. (2009), "The servitization of manufacturing", Journal of Manufacturing Technology Management, Vol. 20, 10.1108/17410380910960984.

Cavalieri, S. and Pezzotta, G. (2012), "Product-service systems engineering: State of the art and research challenges", Computers in Industry, Vol. 63, 10.1016/j.compind.2012.02.006.

Cheung, J., Scanlan, J., Wong, J., Forrester, J., Eres, H., Collopy, P., Hollingsworth, P., Wiseall, S. and Briceno, S. (2012), "Application of value-driven design to commercial aeroengine systems", Journal of Aircraft, Vol. 49, 10.2514/1.C031319.

Faisandier, A. (2012), Systems Opportunities and Requirements, Vol. 2, Sinergy'Com.

Gebauer, H., Fleisch, E. and Friedli, T. (2005), "Overcoming the service paradox in manufacturing companies", European Management Journal, Vol. 23, 10.1016/j.emj.2004.12.006.

Kuijken, B., Gemser, G. andWijnberg, N.M. (2017), "Effective product-service systems: A value-based framework", Industrial Marketing Management, Vol. 60, 10.1016/j.indmarman.2016.04.013.

Lemon, M. and Coley, F.J.S. (2008), "Supporting multiple stakeholders in the design of more sustainable and systemic solutions: The case of the sustainable sports car", Design Principles and Practices: An International Journal-Annual Review, Vol. 2, 10.18848/1833-1874/CGP/v02i02/38278.

Neely, A., Benedetinni, O. and Visnjic, I. (2011), "The servitization of manufacturing: Further evidence", .

Petersen, K., Feldt, R., Mujtaba, S. and Mattsson, M. (2008), "Systematic mapping studies in software engineering",

Sotelo, K.G., Baron, C., Esteban, P., Estrada, C.G. and de J. Laredo Velázquez, L. (2018), "How to find non-functional requirements in system developments", IFAC-PapersOnLine, Vol. 51, 10.1016/j.ifacol.2018.08.272.

Tukker, A. and Tischner, U. (2017), New Business Old Europe: Product-Service Development, Competitiveness and Sustainability, Greenleaf Publishing.

Ulrich, K. and Eppinger, S. (2011), Product Design and Development, McGraw-Hill Education, 5 edition. 


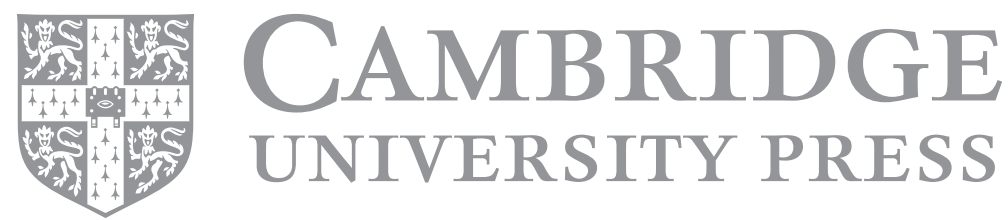

